Draft Version April 10, 2018

Preprint typeset using $\mathrm{LAT}_{\mathrm{E}} \mathrm{X}$ style emulateapj v. 04/17/13

\title{
DISCOVERY OF A TIDAL DWARF GALAXY IN THE LEO TRIPLET
}

\author{
B. NIKIEL-WROCZYŃSKI \\ Obserwatorium Astronomiczne Uniwersytetu Jagiellońskiego, ul. Orla 171, 30-244 Kraków, Poland \\ M. SOIDA \\ Obserwatorium Astronomiczne Uniwersytetu Jagiellońskiego, ul. Orla 171, 30-244 Kraków, Poland \\ D.J. BOMANS \\ Astronomisches Institut, Ruhr-Universität Bochum, Universitätsstrasse 150, 44801 Bochum, Germany \\ M. URBANIK \\ Obserwatorium Astronomiczne Uniwersytetu Jagiellońskiego, ul. Orla 171, 30-244 Kraków, Poland \\ (Received; Revised; Accepted) \\ Draft version April 10, 2018
}

\begin{abstract}
We report discovery of a dwarf galaxy in the Leo Triplet. Analysis of the neutral hydrogen distribution shows that it rotates independently of the tidal tail of NGC 3628, with a radial velocity gradient of $35-40 \mathrm{~km} \mathrm{~s}^{-1}$ over approximately $13 \mathrm{kpc}$. The galaxy has a very high neutral gas content, explaining large part of its total dynamic mass - suggesting a small dark matter content. As it is located at the tip of the gaseous tail, this strongly suggests its tidal origin. Should it be the case, it would be one of the most confident and closest (to the Milky Way) detections of a tidal dwarf galaxy and, at the same time, a most detached from its parent galaxy $(\approx 140 \mathrm{kpc})$ object of this type.
\end{abstract}

Keywords: Galaxies: groups: Arp 317, Leo Triplet - Galaxies: interactions - Intergalactic medium

\section{INTRODUCTION}

The idea of dwarf objects forming from the tidal debris left by galaxy mergers was first proposed by Zwicky (1956), who suggested that interactions in systems of multiple galaxies can lead to an ejection of the tidal material and formation of an intergalactic structure, possibly even a dwarf galaxy. However, the "recycled" galaxies did not achieve much attention, apart from a symposium talk by Schweizer (1978). The first object of this type was discovered by Mirabel et al. (1992), who presented a photometric study of the Antennae galaxies, showing a tidal dwarf galaxy (TDG) formed from the collisional debris. Since then, many similar objects have been detected - see eg. Brinks et al. (2004), or Duc et al. (2007). Recently, Kaviraj et al. (2012) presented a study of a sample of 405 nearby TDG candidates, conducting a statistical analysis of their properties. Tidal dwarf galaxy candidates have been found in the Local Volume (within $11 \mathrm{Mpc}-$ Hunter et al. 2000), too. The M81 group hosts some of the closest examples of the TDGs. Small distance allowed to use the HST-based colour-magnitude diagrams (Makarova et al. 2002) to analyse the star formation history of the TDG candidates and search for additional signs of the tidal origin.

What makes the TDGs especially interesting is their mass composition. Whereas "normal" galaxies consist mostly of the dark matter (DM), TDGs do not; velocity

iwan@oa.uj.edu.pl

soida@oa.uj.edu.pl

bomans@astro.rub.de

urb@oa.uj.edu.pl of the DM particles in the galactic halo is much higher than the escape velocity of a TDG (Bournaud 2010), so they are not kinematically bound to it. Hence, such systems consist usually of the baryonic matter only. Additionally, as they are formed in the outer parts of the galactic disks, their metallicity is higher than in the nontidal dwarfs.

Only several TDGs were estimated to be heavy enough to contain significant non-baryonic fraction, but usual estimates suggest DM content at most similar to the baryonic mass - far below the typical order of magnitude of difference in non-tidal dwarf systems (Bournaud 2010). Lack of the DM content and specific environment cause the evolution of the TDGs to be different from that of typical field galaxies, still to be studied and described. With the low dark matter content TDGs should also be more susceptible to the formation of galactic outflows driven by strong star formation. Alternately, different mass distribution may lead to a lower overall star formation and therefore to a low surface brightness nature of evolved TDG.

TDGs are interesting not only because of their mass composition, but also because of their influence on the intergalactic environment. Tidal debris can interact with other group members, like in the case of the Leo Triplet galaxy NGC 3627, known for its unusual magnetic field morphology (Soida et al. 2001). Recently, Weżgowiec et al. (2012) suggested these peculiarities could be a result of a past collision with a dwarf galaxy. Thus, TDGs might play an important role in the further evolution of their progenitors.

Galaxy systems with massive tidal tails and/or rings 
constitute favourable objects to search for the TDG candidates. One of the best examples of such objects is the Leo Triplet, a nearby group of galaxies, known for a large tidal plume extending eastwards from NGC 3628. Originally described by Zwicky (1956), the plume was later confirmed by photographic observations by Kormendy\&Bahcall (1974). Neutral hydrogen studies by Rots (1978) and Haynes et al. (1979) revealed a thick Hi structure, longer and wider than its optical counterpart. A detailed analysis of the Hi distribution (Stierwalt et al. 2009) suggested numerous candidates for the nontidal dwarf satellites.

Recently, Nikiel-Wroczyński et al. (2013) presented a study of the magnetic field in the Triplet. The authors suggested that the HI clump at the tip of the tidal tail could be a TDG. However, as pointed out in most of the TDG studies (see eg. Kaviraj et al. 2012) determination if a candidate is self-gravitating (galaxy), or rather a larger part of the tidal debris (that will never become a self-bound, independent object) is crucial.

In this paper we use the archive neutral hydrogen and optical data to show that the velocity field of the TDG candidate detected in the Leo Triplet exhibits a velocity gradient and has a faint optical counterpart. These findings strongly support the idea of its independent rotation, thus confirming its identification as a galaxy.

\section{OBSERVATIONS AND DATA REDUCTION}

\subsection{Neutral hydrogen observations}

The $1.41 \mathrm{GHz}$ spectral data, made with the Very Large Array (VLA) of the National Radio Astronomy Observatory (NRAO) 1 in the D-array configuration, were taken from the NRAO Data Archive (Project AB1074, PI: A.Bolatto). Two IFs were set, first at $1.41527 \mathrm{GHz}$, second at $1.41761 \mathrm{GHz}$, each with a bandwidth of $3.1 \mathrm{MHz}$. The corresponding velocity range is $269-1511 \mathrm{~km} \mathrm{~s}^{-1}$. The total bandwidth of the observations is $5.5 \mathrm{MHz}$ and the central frequency of the final data is $1.41645 \mathrm{GHz}$. We used only these fields that contained the TDG candidate. Datasets for each of the pointings used were reduced following the standard spectral line data calibration procedure in the Astronomical Image Processing System (AIPS). The maps were Briggs weighted to detect faint, extended emission. The final angular resolution is $80^{\prime \prime} \times 80^{\prime \prime}$. Assuming the distance to NGC 3628 of $12.15 \mathrm{Mpc}$ (median distance calculated from the TullyFisher-determined values from the NASA Extragalactic Database), this yields the linear resolution of our maps of some $4.7 \mathrm{kpc}$. The velocity resolution is $20.7 \mathrm{~km} \mathrm{~s}^{-1}$ and the r.m.s. noise level $(\sigma)$ of the final maps is approximately $0.63 \mathrm{mJy}$. This corresponds to the brightness temperature of $0.10 \mathrm{~K}$ and $\mathrm{HI}$ column density threshold of $6.9 \times 10^{19} \mathrm{~cm}^{-2}$ (per spectral channel).

\subsection{Optical observations}

A search for an optical counterpart requires sensitive optical and/or near-infrared data. We checked the Sloan Digital Sky Survey (SDSS) DR7 data (Abazajian et al. 2009) in all filters ( $u^{\prime}, g^{\prime}, r^{\prime}, i^{\prime}$, and $\left.z^{\prime}\right)$ individually. A very low surface brightness structure is present in the $g$

1 NRAO is a facility of National Science Foundation operated under cooperative agreement by Associated Universities, Inc.

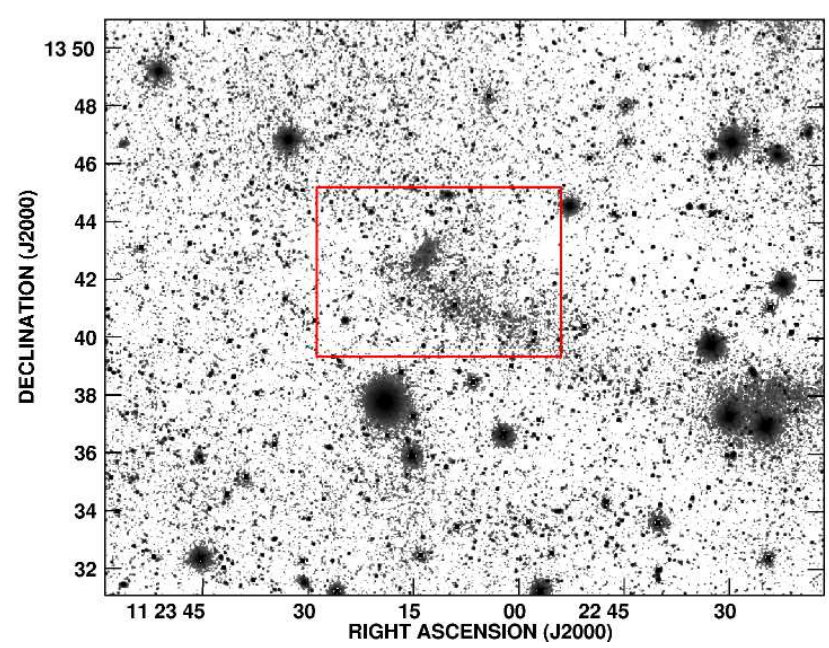

Figure 1. The processed stack of the SDSS $g$ ', r', and i' bands. The faint concentration contained in the central frame is the LeoTDG.

image. To improve on this tentative detection we applied our stacking/filtering procedure, which increases the detectability of very low surface brightness structures (see Miskolczi et al. 2011, for details).

The resulting processed stack of the images in the three most sensitive SDSS filter bands $g^{\prime}, r^{\prime}$, and $i$ 'is presented in Fig. 1

\section{RESULTS}

\subsection{Optical emission distribution}

The processed SDSS image stack (Fig. 1) shows a faint extended region at the position of the Hi plume (diffuse, low surface brightness patch at R.A. $2000=11^{\mathrm{h}} 23^{\mathrm{m}} 15^{\mathrm{s}}$, Dec $_{2000}=13^{\circ} 43^{\prime} 15^{\prime \prime}$ ) and a (fainter) structure elongated along the $\mathrm{E}-\mathrm{W}$ direction. This structure is also visible in a widefield image showing the tail of NGC 3628, provided to us by S. Mandel (reproduced in Fig. 4 in Miskolczi et al. 2011).

The detected patch shows an exponential brightness profile with a central surface brightness of $25.2 \mathrm{mag}$ $\operatorname{sqarcsec}^{-1}$ ( $g$ ' filter) and a scale length of $120^{\prime \prime} \times 60^{\prime \prime}$ $(7 \times 3.5 \mathrm{kpc})$. The total brightness of the structure is $17.1^{m}\left(16.65^{m}\right.$ in the $r^{\prime}$ filter $)$. This means that $g^{\prime}-r^{\prime}$ is $0.45^{\mathrm{m}}$. Using the conversion factors by Jester et al. (2005), these translate to an apparent B-band brightness $m_{\mathrm{B}}=17.45^{m}, \mathrm{~B}-\mathrm{V}$ of $0.615^{m}$, and central surface brightness $\mu_{\mathrm{B}}$ of $25.55^{m}\left(\mu_{\mathrm{V}}=24.93^{m}\right)$. The distance modulus is 30.42 , yielding the absolute B-band magnitude $\mathrm{M}_{\mathrm{B}}$ of $-12.97^{\mathrm{m}}$.

We also check the colour of the tidal tail at two separate positions, one closer $\left(\approx 2^{\prime}\right.$ to the SW from the TDG), one more distant $\left(\approx 13^{\prime}\right.$ to the SW from the TDG). The surface brightness of the more distant position in the tidal arm is comparable to that of the TDG, while the position closer to the TDG is fainter, which unfortunately limits the accuracy of the measurement. Measuring the TDG and both regions in the stream with different methods of background determination implied that the uncertainty of the colour measurements of each of these structures is at least $0.1 \mathrm{mag}$. With that in mind, the colours of both the distant stream clump ( $g$ '$\left.r^{\prime}=0.3\right)$ and the fainter, closer one $\left(g^{\prime}-r^{\prime}=0.4\right)$ are the same as the colour of the TDG itself. With the data at 


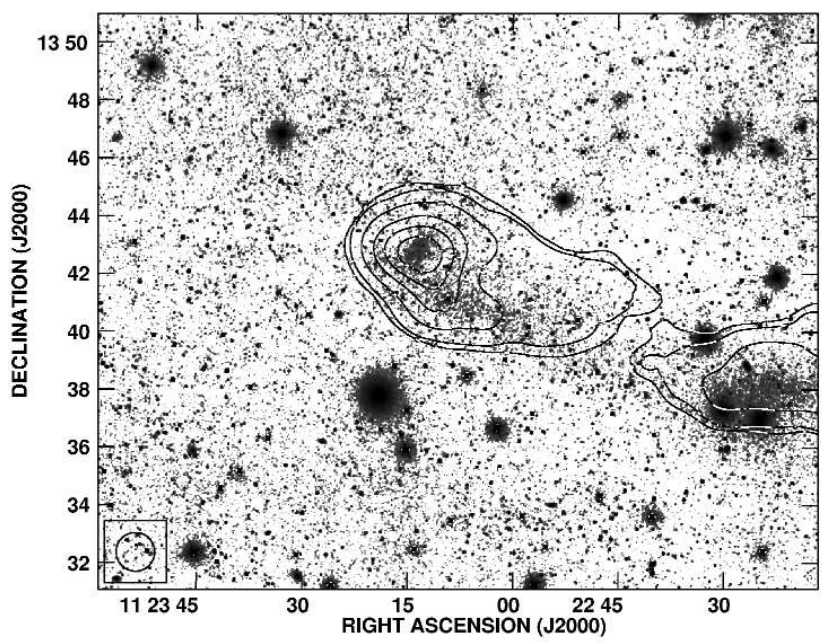

Figure 2. Contours of the zeroth moment of the Hi distribution from the VLA overlaid on a multi-band $\left(g^{\prime}, r^{\prime}\right.$, and $\left.i^{\prime}\right)$ image stack from the SDSS. The contour levels are $5,10,25,50,75 \times 30 \mathrm{Jy} /$ beam $\times \mathrm{m} / \mathrm{s}$. The angular resolution of the radio data is 80 arcsec.

hand, any difference of colour between the stellar population mix in the TDG and the two analysed regions in the stream remains within the uncertainties. A detailed analysis of the stellar populations of the TDG and of the tidal stream must await much better data.

\subsection{Neutral hydrogen distribution}

Figure 2 presents the Hi total intensity (zeroth moment) map of the TDG candidate. It shows a luminous, well-resolved source of approximately ellipsoidal shape of a major axis of $300^{\prime \prime}$ and a minor axis of $275^{\prime \prime}$ with a position angle of $35^{\circ}$. This corresponds to a linear size of $17.5 \times 16 \mathrm{kpc}$. The total intensity is $\approx 9.0 \pm 0.5 \mathrm{Jy} \mathrm{km} \mathrm{s}^{-1}$. The neutral hydrogen data have clear counterparts in the optical regime. Both optical and HI emitting media are connected to the tidal tail.

The first moment (velocity) map is shown in Fig. 3. The velocity gradient runs from the northern (approaching) to the southern (receding) side, where it sinks into the tidal plume. The measured values of the radial velocity range from $860-900 \mathrm{~km} \mathrm{~s}^{-1}$. The tail's velocity is somewhat higher, with a mean of $910 \pm 5 \mathrm{kms}^{-1}$. There is no observable trend along the $\mathrm{E}-\mathrm{W}$ direction. As the velocities of the dwarf system and the tail are different, it appears likely that the dwarf is not bound to the tail. This identifies the dwarf as a separate object, which is self-gravitating - thus, a galaxy. This claim is also supported by the morphology of the tidal tail, which bends strongly in the direction of the TDG candidate in its close vicinity. Such a behaviour suggests that the TDG's gravitational influence on the tail is higher than expected for the internal gravity of the tail. This makes the existence of a self-gravitating object in the tip of the tidal tail even more likely.

To illustrate the separation of the two components we made a contour plot of six channels in which the tail and/or the dwarf system is visible. This map, included as Fig. 4. shows that the tidal tail and the dwarf galaxy are separated and there is at least one channel that only one of them is solely visible.

\section{DISCUSSION}

\subsection{Stellar mass and age}

Due to the very low surface brightness, the SDSS data do not allow to make a detailed fit to the spectral emission distribution (SED) that could be used to derive the star formation history and mass of the Leo-TDG. Still, it is possible to estimate some information from the photometry. Using the scaling relation from Bell et al. (2003), we can use $g$ ' and $r^{\prime}$ magnitudes and the resulting colour to get an estimate of the stellar mass. With $g^{\prime}=17.1^{m}, r^{\prime}=16.65^{m}, g^{\prime}-r^{\prime}=0.45^{m}$, and the values in Table 7 of Bell et al. (2003) we calculate the $\mathrm{M} / \mathrm{L}$ ratio $=3.12$. With the measured $\mathrm{L}_{r^{\prime}}$ of $2.39 \times 10^{7}$ $\mathrm{L}_{\odot}$ this results in a stellar mass of $7.4 \times 10^{7} \mathrm{M}_{\odot}$.

A rough limit for the age of the dominant stellar population can be derived from comparison with the model integrated spectra. Assuming that the dwarf has at least some more or less recent star formation (given its large Hi mass), we decided to use the STARBURST99 code (Leitherer et al. (1999; 2010); Vazquez et al. (2005)) to model basic properties of the stellar population of Leo-TDG. Independently of the assumed metallicity (2 times solar to $1 / 20$ solar) and star formation law (continous or instantanous), for the measured $\mathrm{B}-\mathrm{V}=0.62$ we get a lower age limit of $1 \mathrm{Gyr}$ (which is the limit of the published models). If we assume a moderate internal reddening of $0.3 \mathrm{mag}$, the age limits are from $3 \times 10^{8}$ yrs for a star formation burst and solar metallicity to $\approx 10^{9}$ yrs for $20 \%$ of the solar metallicity. Obviously, while being relatively blue, the majority of the stars formed significantly more than $10^{8}$ years ago. For a more detailed analysis much better photometry is required.

As estimated by Rots (1978), the closest encounter between NGC 3627 and NGC 3628 may have happened $\approx 8 \times 10^{8}$ yrs ago. Thus most of the stars in Leo-TDG (and probably the tidal dwarf itself) had to be formed shortly after the aforementioned collision of these galaxies. It is not likely that these stars formed in NGC 3628 and have been later dragged away, as the distance from the parent object is very large.

\subsection{Gas content}

The gas mass of the Leo-TDG was estimated assuming $\mathrm{M}_{\mathrm{H}_{\mathrm{I}}}\left[\mathrm{M}_{\odot}\right]=2.36 \times 10^{5} D_{\mathrm{Mpc}}^{2} \int S_{\nu} \mathrm{d} \nu$, where $\mathrm{S}_{\nu} \mathrm{d} \nu$ is in $\mathrm{Jy} \times \mathrm{km} \mathrm{s}^{-1}$ (van Gorkom et al. 1986). Using the distance of $12.15 \mathrm{Mpc}$ and total flux of $9.0 \pm 0.5 \mathrm{Jy} \mathrm{km} \mathrm{s}^{-1}$ (see Sect. 3.2), we obtained the total mass of the neutral hydrogen $\mathrm{M}_{\mathrm{H}_{\mathrm{I}}}=3.0-3.3 \times 10^{8} \mathrm{M}_{\odot}$. It is somewhat lower than the results from Stierwalt et al. (2009), but still of the same order of magnitude. The differences are most likely caused by the larger beamsize of the Arecibo telescope used by the authors of the former study, which causes confusion of the emission from the dwarf candidate by that from the tail.

\subsection{Mass-to-light ratio and the total mass}

The dynamical mass $M_{D Y N}$ of Leo-TDG can be derived from the rotational velocity at a given radius. For Leo-TDG, the radial velocity (not corrected for the inclination) gradient is about $35-40 \mathrm{~km} \mathrm{~s}^{-1}$ over some $13 \mathrm{kpc}$ (with the tail contribution subtracted). The neutral hydrogen data do not allow to reliably estimate the turbulent component. Therefore, we decided to 


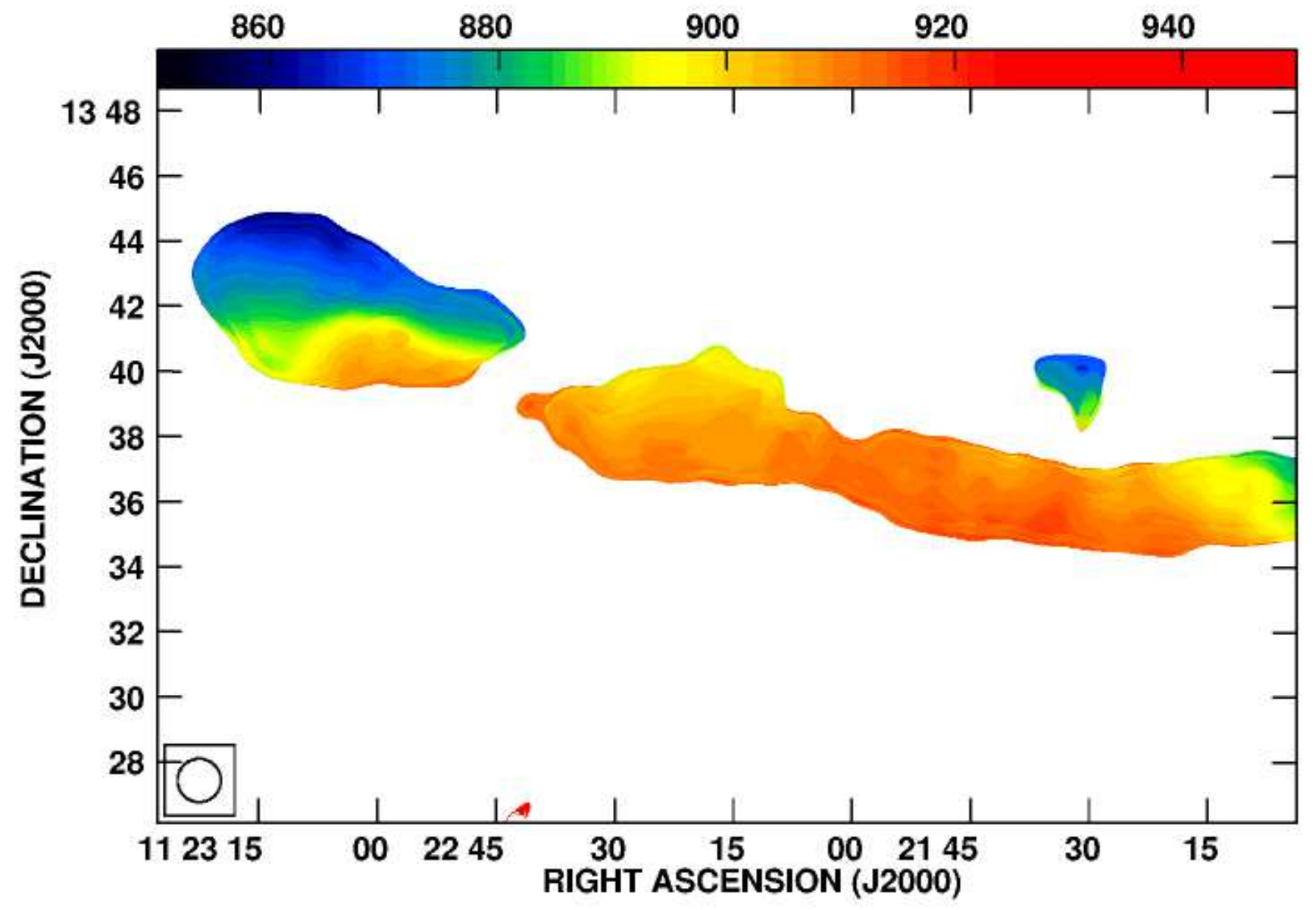

Figure 3. The Hi velocity distribution map made from the VLA observations. The colours represent gas species of velocities ranging from 850 (dark navy) to $950 \mathrm{~km} \mathrm{~s}^{-1}$ (red). The angular resolution is $80 \operatorname{arcsec}$, and the spectral resolution is $20.7 \mathrm{~km} \mathrm{~s}^{-1}$.

use a conservative assumption of $10 \mathrm{kms}^{-1}$ for a 1dimensional turbulent contribution. If this is used to calculate the dynamical mass, one can obtain a total mass of some $7.9 \times 10^{8} \mathrm{M}_{\odot}$. With the inclination unknown, this value can be treated as a lower limit of the dynamical mass. For a reasonable inclination of about $60^{\circ}$ (based on the elongation of the the optical and $\mathrm{HI}$ shape of the dwarf), the total dynamical mass would rise to $\mathrm{M}_{\mathrm{DYN}}=1.41 \times 10^{9} \mathrm{M}_{\odot}$. It should be strongly indicated here, that the dynamical mass estimate comes with a large uncertainty. As the dependence of the dynamical mass on the (unknown) inclination is given by is given by $M_{D Y N} \propto 1 / \sin (i)^{2}$, the dynamical mass would largely increase if only Leo-TDG is a more faceon-orientated system. In general, estimation of masses of the dwarf galaxies and their distributions is a complicated issue, as even if the inclination estimate is proper to some extent, the question of the finite disk thickness persists (Rhee et al. 2004). The total baryonic content of Leo-TDG can be calculated as a sum of the stellar mass $\left(7.4 \times 10^{7} \mathrm{M}_{\odot}\right)$ and gaseous component. Assuming a modest estimate of the molecular gas mass of $10-30 \%$ of the HI mass (as $\mathrm{M}_{\mathrm{H}_{2}} / \mathrm{M}_{\mathrm{H}_{\mathrm{I}}}$ for NGC 3628 is equal to $\approx 20 \%$, Obreschkow\&Rawlings 2009), the total gas mass would be around $3.3-4.3 \times 10^{8} \mathrm{M}_{\odot}$, so the total baryonic content $\mathrm{M}_{\mathrm{BAR}}$ is $4.0-5.0 \times 10^{8} \mathrm{M}_{\odot}$.

Estimate of $\mathrm{M}_{\mathrm{DYN}} / \mathrm{L}_{\mathrm{B}}$ can also be derived. $\mathrm{L}_{\mathrm{B}}\left[\mathrm{L}_{\odot}\right]$ is equal to $10^{-0.4 \times\left(\mathrm{M}-\mathrm{M}_{\odot}\right)}$. The B-band magnitude of the Sun is equal to 5.47 (Cox 1998). This yields the total Bband luminosity of $2.4 \times 10^{7} \mathrm{~L}_{\odot}$. The $\mathrm{M}_{\mathrm{DYN}} / \mathrm{L}_{\mathrm{B}}$ is then $33-59$, and $\mathrm{M}_{\mathrm{H}_{\mathrm{I}}} / \mathrm{L}_{\mathrm{B}}$ is $12-14$.

\subsection{Magnetic field}

The resolutions used in our previous study (NikielWroczyński et al. 2013) $-4^{\prime} .3$ in the radio continuum and $3^{\prime} .5$ in the Hi data of Stierwalt et al. (2009) gave no grounds to reject the coincidence of the HI and radio continuum-emitting regions. There were also no reliable optical images available. All this was suggestive for the existence of the magnetic field in Leo-TDG.

With almost 4 times smaller beam of the Hi data analysed in this work and using our optical image we could state that the radio peak is shifted by approximately 1 ' westwards from the neutral gas peak and seems to be located outside the optical emission. A large fraction of the radio continuum emission may be thus due to a background source. In the light of the new data we need to revise the estimate of the magnetic field strength. Setting the upper limit to the radio emission of $3.0 \mathrm{mJy} / \mathrm{beam}$ at the position of gaseous and optical feature implies the total magnetic field in the TDG to be $\mathrm{B}_{\mathrm{TOT}} \leq 2.8 \mu \mathrm{G}$. The magnetic and cosmic-ray energy density amounts therefore to $\mathrm{P}_{\mathrm{B}+\mathrm{CR}} \leq 6.8 \times 10^{-13} \mathrm{erg} \cdot \mathrm{cm}^{-3}$.

\subsection{TDG or a non-tidal, LSB galaxy?}




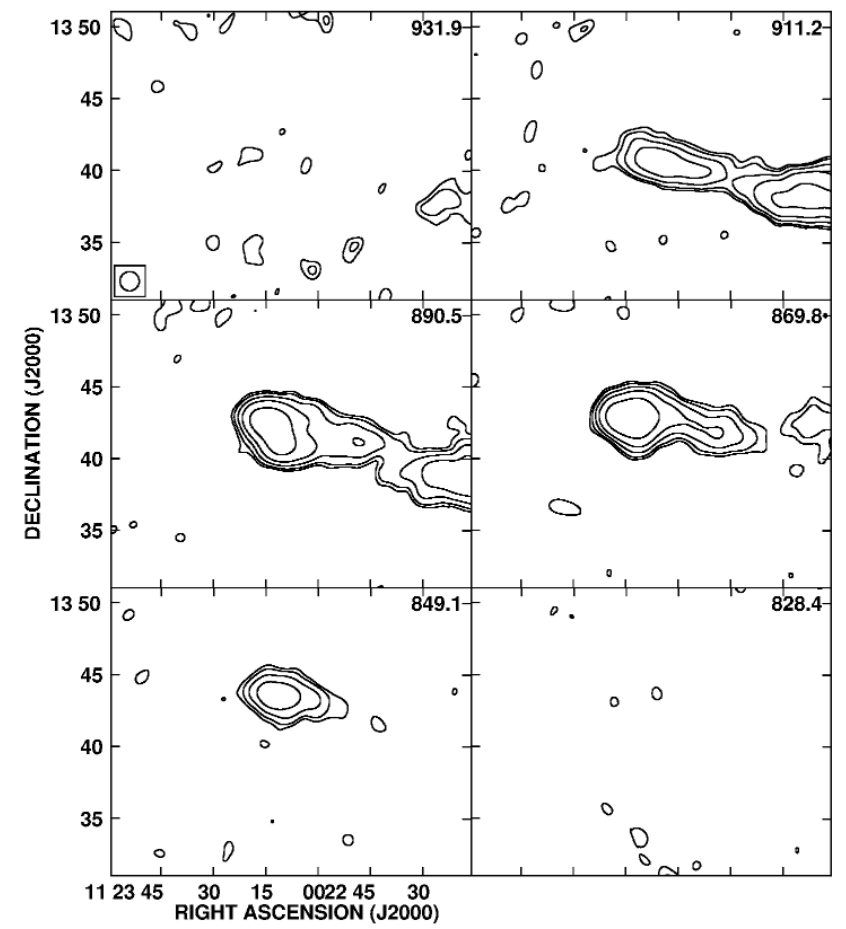

Figure 4. Six-channel map of Leo-TDG. Central velocities (in $\mathrm{km} \mathrm{s}^{-1}$ ) of each of the channels are written in the upper right corner of each of the planes. The angular resolution is $80 \operatorname{arcsec}$; the spectral resolution is $20.7 \mathrm{~km} \mathrm{~s}^{-1}$.

Leo-TDG shares many of its characteristics with the TDGs (Kaviraj et al. 2012). It is rather bluer than its supposed progenitor $(0.65$ compared to 0.8 for NGC 3628, Paturel et al. 2003), it is located exactly at the tip of the tidal tail, and has mass of some $10^{8} \mathrm{M}_{\odot}$, typical for such objects. On the other hand, if identified as a TDG, the discussed object would be the tidal dwarf most distant from its parent object, with a calculated separation of some $140-150 \mathrm{kpc}$, while $95 \%$ of the TDG candidates do not lie more than $20 \mathrm{kpc}$ from their progenitors (Kaviraj et al. 2012). Compared to the statistical sample, Leo-TDG is dim, as it contains less stars than typical TDG candidates. Among the most distinct features of this galaxy are its low surface brightness $\left(\mu_{\mathrm{B}}=25.55\right)$ and very high abundance of the neutral gas. Because of that, we compare its properties not only with the TDGs, but also Low Surface Brightness (LSB) galaxies. We decided to take a galaxy (F563-1) from the samples collected by de Blok et al. (1995; 1996) and the "dark" LSB NGC 3741 (Begum et al. 2005; 2008). As a comparison TDG, we have chosen the "old TDG" VCC 2062 (Duc et al. 2007). The data for the selected objects (TDGs and LSBs) are shown in Table 1.

The table clearly shows that the detected galaxy shares parameters of both TDGs and LSBs. In fact, it is not the only one dwarf system that is considered to be either a TDG or an LSB - likewise is the VCC 2062 in the Virgo Cluster (Duc et al.2007). Both galaxies share similar characteristics: they are dim, low-mass systems with low surface brightness. They have a significant neutral hydrogen halo, showing signs of rotation, independent from the tidal arc movement. The velocity gradients are - to the limits of inclination - similar. However, the sizes of the Hi haloes are different, as the one of VCC 2062 is just $4.2 \mathrm{kpc}$ - approximately three times smaller than that of Leo-TDG. The main difference between the LeoTDG and non-tidal LSBs is the dominance of the gas content in the former one. In most of the LSBs, gas is not a dominant component: $\mathrm{M}_{\mathrm{H}_{\mathrm{I}}} / \mathrm{L}_{\mathrm{B}}$ is close to 1 , and $\mathrm{M}_{\mathrm{H}_{\mathrm{I}}} / \mathrm{M}_{\mathrm{DYN}}$ is less than $10 \%$ (de Blok 1995). In case of Leo-TDG, HI dominates over other fractions, manifesting as a very high $\mathrm{M}_{\mathrm{H}_{\mathrm{I}}} / \mathrm{L}_{\mathrm{B}}(12-14)$ and $\mathrm{M}_{\mathrm{H}_{\mathrm{I}}} / \mathrm{M}_{\mathrm{DYN}}$ of $25-$ $55 \%$. Such high neutral gas content causes $\mathrm{M}_{\mathrm{DYN}} / \mathrm{L}_{\mathrm{B}}$ to be higher than in VCC 2062, while $\mathrm{M}_{\mathrm{DYN}} / \mathrm{M}_{\mathrm{BAR}}$ is very similar. As shown by de Blok (1996), non-tidal LSB's have rather high $M_{D Y N} / M_{H_{I}}$ ratios, which is different from the case of the gas-dominated Leo-TDG. $\mathrm{M}_{\mathrm{DYN}} /$ $\mathrm{M}_{\mathrm{BAR}}$ of the non-tidal galaxies are also much higher than $1.6-3.5$ estimated for Leo-TDG (Bournaud et al. 2010). All these features strongly favour scenario of the tidal origin of Leo-TDG.

\section{SUMMARY}

We used the archive VLA Hi spectral data and the SDSS optical observations of the TDG candidate in the Leo Triplet of galaxies. We obtained maps of the zeroth and first kinematic moments of the Hi content as well as the distribution of the visible light in the SDSS $g^{\prime}, r^{\prime}, i$, bands, yielding the following results:

- There is a massive, star-forming HI clump at the tip of the tidal tail of the Leo Triplet.

- Velocity field shows a non-negligible gradient (approximately $35-40 \mathrm{~km} \mathrm{~s}^{-1}$ over $13 \mathrm{kpc}$ ) along the $\mathrm{N}-\mathrm{S}$ (declination) axis, strongly supporting that the detected clump is a self-gravitating, tidal dwarf galaxy (TDG).

- The dwarf galaxy is unusually distant from its host galaxy (approximately $140-150 \mathrm{kpc}$ ), which is more than seven times further than the typical values.

- The optical counterpart has been detected in the SDSS $g$ ' and $r$ ' bands. The apparent B magnitude $m_{\mathrm{B}}$ is $17.45^{m}, \mathrm{~B}-\mathrm{V}$ is $0.615^{m}$, and the central surface brightness $\mu_{\mathrm{B}}$ reaches $25.55^{\mathrm{m}}$. The absolute $\mathrm{B}$-band magnitude is $-12.97^{\mathrm{m}}$.

- The total HI mass of the clump $\mathrm{M}_{\mathrm{H}_{\mathrm{I}}}$ is $3.0-3.3$ $\times 10^{8} \mathrm{M}_{\odot}$. The stellar content is about $7.4 \times 10^{7} \mathrm{M}_{\odot}$.

- The stellar population age is $3 \times 10^{8}-10^{9}$ years. This means that, despite rather blue colour, most of the stars are relatively old.

- The estimated dynamical mass is just $1.6-3.5$ times the baryonic mass. This means that the dark matter content plays much less sound role than in the non-tidal dwarf galaxies. Estimated $\mathrm{M}_{\mathrm{DYN}} / \mathrm{L}_{\mathrm{B}}$ is $33-59$, but this value is mostly due to high $\mathrm{M}_{\mathrm{H}_{\mathrm{I}}} / \mathrm{L}_{\mathrm{B}}(12-14)$. 
Table 1

Parameters of Leo-TDG compared to TDG's and LSB's

\begin{tabular}{ccccc}
\hline \hline Name & Leo-TDG & F563-1 & VCC 2062 & NGC 3741 \\
Type & TDG & LSB galaxy & TDG & LSB (very dark) \\
Opt. size $[\mathrm{kpc}]$ & 7.5 & $3.4^{1}$ & $0.7^{1}$ & 1.7 \\
Hi size $[\mathrm{kpc}]$ & 13 & $16^{1}$ & 4.2 & 14.6 \\
$\mu_{\mathrm{B}}\left[\mathrm{mag}_{\mathrm{masec}}{ }^{-1}\right]$ & 25.55 & 23.79 & 24.85 & 24.91 \\
$\mathrm{~B}-\mathrm{V}$ & 0.615 & 0.58 & 0.35 & $0.36^{2}$ \\
Total mass $\left[\mathrm{M}_{\odot}\right]$ & $7.9-14.1 \times 10^{8}$ & $3.9 \times 10^{10}$ & $3-4 \times 10^{8}$ & $4 \times 10^{9}$ \\
Gas content $\left[\mathrm{M}_{\odot}\right]$ & $3.3-4.3 \times 10^{8}$ & $1.5 \times 10^{9}$ & $0.8 \times 10^{8}$ & $1.6 \times 10^{8}$ \\
Stellar content $\left[\mathrm{M}_{\odot}\right]$ & $7.4 \times 10^{7}$ & $2.3 \times 10^{8}$ & $0.2-0.7 \times 10^{8}$ & $1.4 \times 10^{7}$ \\
$\mathrm{M}_{\mathrm{H}_{\mathrm{I}}} / \mathrm{L}_{B}$ & $12-14$ & 2.06 & 3 & 6.26 \\
$\mathrm{M}_{\mathrm{B}}$ & -12.97 & -16.7 & -13 & -13.13 \\
$\mathrm{M}_{\mathrm{DYN}} / \mathrm{M}_{\mathrm{BAR}}$ & $1.6-3.5$ & $\approx 17^{3}$ & $2-4$ & 24 \\
$\mathrm{M}_{\mathrm{DYN}} / \mathrm{L}_{\mathrm{B}}$ & $33-59$ & 50.1 & $\approx 10$ & 149 \\
\hline
\end{tabular}

${ }^{1}$ Derived from its angular size

${ }^{2}$ From Taylor and Webster (2005)

${ }^{3}$ Derived from the M/L ratio calculated basing on Bell (2003)

- Compared to the similar objects, Leo-TDG is relatively dim, and has a very high fraction of neutral gas, implying a short evolution time and/or low star formation rate since its formation.

We thank the anonymous refferee for valuable comments and suggestions that helped to improve our paper. We acknowledge the usage of the NASA/IPAC Extragalactic Database (NED) which is operated by the Jet Propulsion Laboratory, California Institute of Technology, under contract with the National Aeronautics and Space Administration. This research has made use of NASA's Astrophysics Data System. This research has been supported by the scientific grant a the National Science Centre (NCN), decision no. DEC2011/03/B/ST9/01859. Funding for the SDSS and SDSS-II has been provided by the Alfred P. Sloan Foundation, the Participating Institutions, the National Science Foundation, the U.S. Department of Energy, the National Aeronautics and Space Administration, the Japanese Monbukagakusho, the Max Planck Society, and the Higher Education Funding Council for England. The SDSS Web Site is http://www.sdss.org/.

\section{REFERENCES}

Abazajian, K. N.; Adelman-McCarthy, J. K.; Agüeros, M. A. et al. 2009, ApJS, 182, 543

Bell, E. F; McIntosh, D. H; Katz, N. et al. 2003, ApJS, 149, 289

Begum, A.; Chengalur, J. N.; Karachentsev, I. D.2005, A\&A, 433, 1

Begum, A.; Chengalur, J. N.; Karachentsev, I. D. 2008, MNRAS, 386, 1667

Brinks, E.; Duc, P.-A.; Walter, F.2004, in: Proceedings of the International Astronomical Union, 2004, 532

Bournaud, F. 2010, Advances in Astronomy, 2010

Bournaud, Duc 2008, ApJ, 672, 787
Condon, J. J.; Cotton, W. D.; Greisen, E. W. et al. 1998, ApJ, 115,1693

Allen's astrophysical quantities, 4th ed. Publisher: New York: AIP Press; Springer, 2000. Edited by Arthur N. Cox. ISBN: 0387987460

de Blok, W. J. G.; van der Hulst, J. M.; Bothun, G. D. 1995, MNRAS, 274, 235

de Blok, W. J. G.; McGaugh, S. S.; van der Hulst, J. M. 1996, MNRAS, 283, 218

Duc, P.-A.; Braine, J.; Lisenfeld, U. et al. 2007, A\&A, 475, 187

Duc, P.-A; Bournaud, F.; Brinks, E. 2008, IAU Symposium, 244, 216

Haynes, M. P.; Giovanelli, R.; Roberts, M. S. 1979, ApJ, 229, 83

Hunter, D. A.; Hunsberger, S. D.; Roye, E. W. 2000, ApJ, 542 137

Jester, S.; Schneider, D. P.; Richards, G. T. 2005, AJ, 130, 873

Kaviraj, S.; Darg, D.; Lintott, C. et al. 2012, MNRAS, 419, 70

Kormendy, J.; Bahcall, J. N. 1974, AJ, 79, 671

Leitherer, C.; Schaerer, D.; Goldader, J. D. 1999, ApJS, 123, 3

Leitherer, C; Ortiz Otálvaro, P. A.; Bresolin, F. 2010, ApJS, 189, 309

Makarova, L. N.; Grebel, E. K.; Karachentsev, I. D. et al. 2002, A\&A, 396, 473

Minchin, R.; Davies, J.; Disney, M.et al. 2005, ApJ, 622, 21

Mirabel, I. F.; Dottori, H.; Lutz, D. 1992, A\&A, 256, 19

Miskolczi, A.; Bomans, D. J.; Dettmar, R.-J. 2011, A\&A, 536, A66

Nikiel-Wroczyński, B.; Soida, M.; Urbanik, M. et al. 2013, A\&A, 553, A4

Obreschkow, D.; Rawlings, S. 2009, MNRAS, 394, 1857

Paturel, G.; Petit, C.; Prugniel, Ph. et al. 2003, A\&A, 412, 45

Rhee, G.; Valenzuela, O.; Klypin, A. et al. 2004, ApJ, 617, 1059

Rots, A. H. 1978, AJ, 83, 219

Schweizer, F. 1978. In Structure and Properties of Nearby

Galaxies, ed. E.M. Berkhuijsen, R. Wielebinski, p. 279

Soida, M.; Urbanik, M.; Beck, R. et al. 2001, A\&A, 378, 40

Stierwalt, S.; Haynes, M. P.; Giovanelli, R. et al. 2009, ApJ, 138, 338

Taylor, E. N.; Webster, R. L. 2005, ApJ, 634, 1067

van Gorkom, J. H.; Knapp, G. R.; Raimond, E. et al. 1986, AJ, 91, 791

Vázquez, G. A.; Leitherer, C. 2005, ApJ, 621, 695

Weżgowiec, M.; Soida, M.; Bomans, D. J. 2012, A\&A, 544, 113

Zwicky, F.; 1956, Ergebnisse der Exakten Naturwissenschaften 29, 344 